\title{
Improvement in billets quality from billet caster by roller suspension of secondary cooling zone stiffening
}

\author{
Mariya Androsenko ${ }^{1, *}$,Ekaterina Kulikova ${ }^{1}$, and Olga Osipova ${ }^{1}$ \\ ${ }^{1}$ Nosov Magnitogorsk State Technical University, Magnitogorsk, Russia
}

\begin{abstract}
Presently, continuous casting machines are widely used in metallurgical industry for production of high quality billets. And the maximum economic and resource-saving effect is achieved by the use of continuously casting small-section billets. Proceeding to the continuous casting has tightened the requirements for product quality and the main objective has become to determine the factors influencing the billet quality. It is necessary to design competently a zone of secondary cooling, for improvement of quality of production. Having changed her design and as a result the loadings operating on a zone of secondary cooling will decrease and the rigidity of a design will increase.
\end{abstract}

Billet casters with basic radius of $9 \mathrm{~m}$ were put in operation at OAO "MMK" in 2004. The new casters were designed for production of 100x100, 124x124, 120x150 и 152x170 mm billets. However, in the course of commissioning of three new rolling mills supplied by Danieli, the specialists of MMK have developed a technology for production of 150x150 mm billets. Open stream casting is used for casting of billets of all sizes, shrouded casting is used

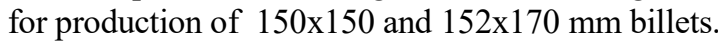

The quality of the billet is determined by internal defects (centre porosity, centerline segregation, crack segregation streamer, bright fringe, edge point contamination, blow hole) and external defects (shell, rhomboidity, scratch, protuberance, concavity, teeming arrest).

Investigation of the available data from sheets with information on distribution of intrashop rejects provided by production control department of OAO "MMK" has shown that the main type of billet defects is non conformance of section geometry.

Different defects of manufactured grades of steel (St2sp, St3sp; SV-08A; 60CA) were analysed. Such geometry defect as "rhomboidity" has turned to have the highest percentage of occurrence in comparison with other defects $(50 \% ; 56 \% ; 28,6 \% ; 45,6 \%)$.

Geometry defects are caused by non-compliance of the following process parameters: 1 ) casting speed (stripping speed) and related water consumption in the secondary cooling zone; 2) casting temperature (temperature of hot metal in a ladle); 3) method of casting (open stream, shrouded); 4) design of the secondary cooling zone.

\footnotetext{
*Corresponding author: manechka.05@mail.ru
} 
Researches to improve casting technology and billet quality have been carried out for several years. It is shown that cracks are formed in billets with rhomboidity exceeding 12 $15 \mathrm{~mm}$ and during the casting of steel with high content of sulphur. As a result the factors have been determined that influence billet shape deterioration as well as measures have been developed to eliminate the defect during the casting. The highest degree of rhomboidity (up to $24 \mathrm{~mm}$ ) is common for casting without support rollers under the mould that are dismantled after shell breakthrough for speed-up of strands restarting.

For billet caster the rhomboidity is in the range from $1 \mathrm{~mm}$ up to $18 \mathrm{~mm}$ with the tendency to decrease every year. In 2009 it was in the range from 1 up to $14 \mathrm{~mm}$. As a result it was concluded that two rows of rollers under the mould secondary cooling zone didn't provide for high quality output due to large distance between roller sections and pour stiffness and it lead to buckling under the ferrostatic pressure of solidifying shell.

In order to reduce loads to elements of roller suspension, to improve stiffness and to decrease contact loads between rollers and billets that cause surface and billet deformation it was proposed to change the suspension design. Three rows of rollers were installed on hangers on the existing base. And the nozzles are evenly distributed in the gaps between the rows.

Results of calculation of bending moments and lateral forces, stress-strain state of billet and roller suspension of old and new construction were used during engineering of a new construction.

In order to determine how the design parameters of the new construction influence the geometry of the billet it is necessary to calculate contact stiffness for both constructions and carry out comparative analysis as criteria of casters runnability are durability, stiffness, wear and heat resistance, accuracy and etc.

From the sources of literature it is known that neglecting of contact stiffness causes inaccurate evaluation of working tool position (tool head, gripper) up to $70 \%$, for example, in screw strap force - up to $50 \%$, in screw strength estimation - up to $30 \%$, in estimation of pressure in tight cylindrical connections - up to $40 \%$.

In the easiest case the capability of linear elastic system or element to resist applied static load is characterised by stiffness of the elastic system or element.

Stiffness is calculated as ratio of component force $P_{y 0}$, directed surface wise and shifting of $y$ in the same direction:

$$
j=\frac{P_{y o}}{y\left(P_{o}\right)}
$$

It is considered that the system is influenced by $P_{y 0}$ and simultaneously by other components $\left(P_{x 0}, P_{z 0}\right)$ of force $P_{o}$. Shifting depends on force $P_{o}$, thus $y\left(P_{o}\right)$. Elastic properties of compound elements of process systems, consisting of several parts, are impossible to determine by one stiffness coefficient as relationship between force and butt joint edge angle (elastic behaviour) is non-linear. To make the calculations easy the nonlinear characteristic in the operational range of the forces is replaced by the linear characteristic, thus:

$$
j=\frac{\Delta P_{y o}}{\Delta y\left(P_{o}\right)}
$$

In general, stiffness, element and system elastic behaviour is determined by calculations or experimentally. As unit stiffness depends on direction and point of force application the researches are carried out in conditions that a closely simulate the real operational conditions of the unit: the force applied to the unit is the same with regards to the force intensity and direction as the constant component force. Loading is performed when the 
unit is in a static state to get static stiffness characteristics. However this parameter doesn't match the real stiffness of the unit in operation.

By analogy with static stiffness $j$ the capability of system or element to resist the applied constant force component $P_{y 0}$ at the given speed of billet transferring is characterised by quasi-static stiffness:

$$
j_{\kappa c m}(n)=\frac{P_{y o}}{y\left(P_{o}, n\right)}
$$

Besides, the dynamic unit stiffness is defined as ratio between

$$
P_{y k} \cdot \cos k \cdot w \omega t
$$

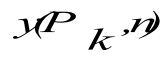

force harmonic component and caused by it shifting:

$$
j_{\text {дин }}=\frac{P_{y k} \cdot \cos k \cdot \omega t}{y\left(P_{k}, n\right)}
$$

where $\omega$ - angular velocity, frequency of force harmonic component, where $k=1$, caused by force change due to shifting (offset) of profile of roller axis.

The shifting of system element is evaluated by angular shifting in radians. In accordance with it the unit of stiffness measurement is changed.

Compliance is used for process calculations for such static conditions as butt joint edge angle, caused by the force equal to 1 , that is the value of the inverse stiffness:

$$
W=\frac{1}{y}=\frac{y}{P_{y}}
$$

The same way the quasi-static compliance $w_{\kappa с m}$ and dynamic compliance $w_{\text {дин. }}$. is calculated.

For rough estimate values $j$ and $w$ that determine properties of statically loaded idle system are used.

On the basis of calculations it can be concluded that new suspension design reduces loads between rollers and the billet. It allows to reduce deformation of the geometry of the billet produced by the caster.

There were problems with the determining of the axes coordinates of the secondary cooling zone rollers at design stage of secondary cooling zone unit. Application for the patent has been made and then the patent [2] for a utility model has been acquired, according to which (Figure 1) axes coordinates for holes for the rollers in plane y forming near $b_{y i}$ and far $b_{y i}^{\prime}$ radial webs with regards to the base are determined by the following relationships:

$$
\begin{gathered}
b_{y i}=i \cdot 1,5 d \\
b_{y i}^{\prime}=\frac{b_{y i} \cdot(2 R+d)}{2 \cdot(R-L)-d}
\end{gathered}
$$

and the axes coordinates of the holes for the rollers along $\mathrm{x}$ forming close $b_{x i}$ and far radial $b_{x i}^{\prime}$ webs with regards to the axis perpendicular to the base and coming from the middle of the strand are determined by the following relationships:

$$
\begin{aligned}
& b_{x i}=\frac{L}{2}+\frac{d}{2}+b_{y i} \cdot \sin \frac{2 \cdot \sin ^{-1} \frac{b_{y i}}{2 \cdot(R-L)-d}}{2} \\
& b_{x i}^{\prime}=\frac{L}{2}+\frac{d}{2}-b_{y i}^{\prime} \cdot \operatorname{tg} \frac{2 \cdot \sin ^{-1} \frac{b_{y i}}{2 \cdot(R-L)-d}}{2}
\end{aligned}
$$


where $i$ - section consecutive number;

$d$ - roll diameter;

$R$ - casting machine radius;

$L-$ billet size

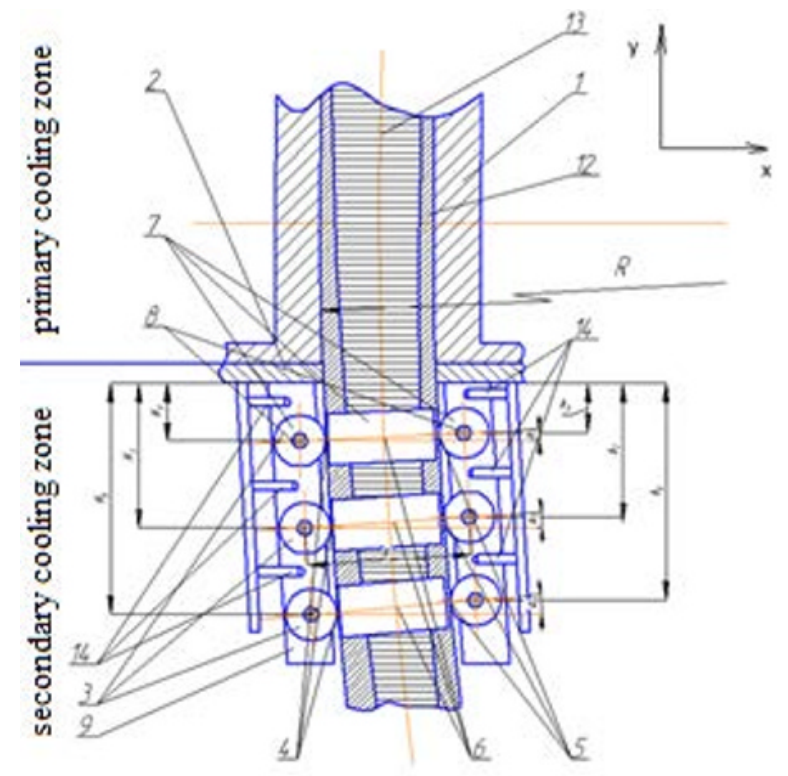

Fig. 1. Construction of secondary cooling zone with three roller sections.

For comparison of the stress-strain state of the ingot emerging from the mould with the old secondary cooling zone (two rows of rollers) with a mould with a new secondary cooling zone (three rows of rollers) corresponding three-dimensional models for the calculation in Ansys Workbench were developed. Thus, the assumption was made: the roller sections were in contact with the ingot for a short period of time (static solution). The above mentioned contact interaction was modelled as elastoplastic body. Figure 2 shows the indicators of the stress-strain state of the construction with two and three roller section of secondary cooling zone.

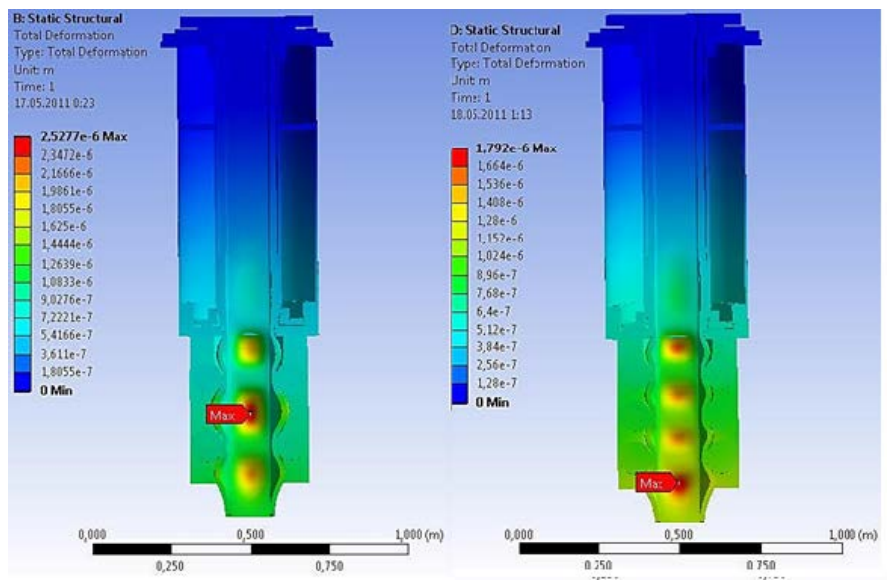

Fig. 2. Indicators of stress-strain state of the construction with two and three roller suspension. 
Analysis of the data shown in Figure 2 proves that the new design is less stressed in comparison with the old one. The roller unit with additional roller section is designed, manufactured and commercially tested at the caster of MSP at OAO "MMK". The billet quality was improved by $15-20 \%$ due to rhomboidity decrease.

\section{References}

1. A.B. Great, et al. Study the causes of distortions Profile graded continuous casting. Foundry processes. Magnitogorsk: GOU VPO "Magnitogorsk State Technical University". - (2008). - Vol. 7. - pp. 152 - 157.

2. The device for maintenance of an ingot in a zone of secondary cooling of MNLZ: Stalemate. The Russian Federation on useful model No. 105849: MPK: B 22 D 1112. Patent holder Magnitogorsk Iron and Steel Works Open joint stock company.-No. 2010144659/02; заяв. 11/1/2010; opub.27.06. 2011; bulletin No. 18

3. M.V. Androsenko, Integral Assessment of Quality Continuous Cast Billet Caster EAFPnm . Eastern European Scientific Journal. AURIS Kommunikations- und VerlagsgesellschaftmbH. - (2014). DOI 10.12851 / EESJ201406

4. S.A. Botnikov, Influence of chemical composition and technology of refining low carbon and medium carbon steel casting parameters on the "MMK".Sciences: 05.16.02: 24.06.09 protected / Botnikov Sergey. - Chelyabinsk, (2009). - 23 pp.

5. A.M. Stolyarov, Improving the efficiency of casting steel high-koproizvoditelnyh "MMK". Magnitogorsk: GOU VPO "MagnitogorskState Technical University", (2009). - 126 pp.

6. M.J. Brovman, Improvement of technology and equipment of continuous casting matire blanks. Kiev: Engineering, 1976.-165 pp.

7. M.V. Androsenko, I. D. Kadoshnikova Quality check and analysis of the concast billets . Heavy engineering industry. (2015) / № 9. P. 30-33. 